\title{
Cardiovascular health in early adulthood predicts the development of coronary heart disease in individuals with type 1 diabetes: 25 year follow-up from the Pittsburgh Epidemiology of Diabetes Complications study
}

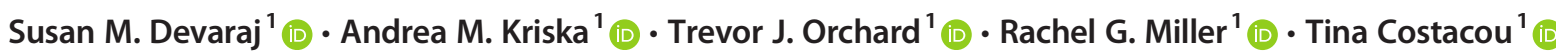

Received: 26 May 2020 / Accepted: 1 October 2020 / Published online: 14 November 2020

(C) Springer-Verlag GmbH Germany, part of Springer Nature 2020

\begin{abstract}
Aims/hypothesis Type 1 diabetes increases CHD risk. We examined the use of the American Heart Association's cardiovascular health metrics (blood pressure, total cholesterol, glucose/ $\mathrm{HbA}_{1 \mathrm{c}}$, BMI, physical activity, diet, smoking) to predict incidence of CHD among individuals with type 1 diabetes, with the hypothesis that a better American Heart Association health metric profile would be associated with lower incident CHD.

Methods Prevalence of the seven cardiovascular health metrics was determined using first and second visits from adult participants (mean age 28.6 years) in the Epidemiology of Diabetes Complications prospective cohort study of childhood-onset type 1 diabetes. An ideal metric score (0-7) was defined as the sum of all metrics within the ideal range, and a total metric score (0-14) was calculated based on poor, intermediate and ideal categories for each metric. Incident CHD development (medical recordconfirmed CHD death, myocardial infarction, revascularisation, ischaemic electrocardiogram changes or Epidemiology of Diabetes Complications physician-determined angina) over 25 years of follow-up was examined by metric scores.

Results Among 435 participants, BMI, blood pressure, total cholesterol and smoking demonstrated the highest prevalence within the ideal range, while diet and $\mathrm{HbA}_{1 \mathrm{c}}$ demonstrated the lowest. During 25 years of follow-up, 177 participants developed CHD. In Cox models, each additional metric within the ideal range was associated with a $19 \%$ lower risk $(p=0.01)$, and each unit increase in total metric score was associated with a $17 \%$ lower risk $(p<0.01)$ of $\mathrm{CHD}$, adjusting for diabetes duration, estimated glomerular filtration rate, albumin excretion rate, triacylglycerols, depression and white blood cell count.

Conclusions/interpretation Among individuals with type 1 diabetes, higher cardiovascular health metric scores were associated with lower risk of incident CHD. The American Heart Association-defined cardiovascular health metrics provide straightforward goals for health promotion that may reduce CHD risk in the type 1 diabetes population.
\end{abstract}

Keywords Cardiac complications · Epidemiology $\cdot$ Exercise $\cdot$ Hypertension $\cdot$ Lipids/lipoproteins $\cdot$ Nutrition and diet $\cdot$ Weight regulation and obesity

\section{Abbreviations}

AHA American Heart Association

BDI Beck Depression Inventory

CACTI Coronary Artery Calcification in Type 1 Diabetes

DBP Diastolic blood pressure

EDC Epidemiology of Diabetes Complications
LS7 Life's Simple Seven

MACE Major adverse cardiovascular events

MET Metabolic equivalent of task

SBP Systolic blood pressure

WBC White blood cell count
Tina Costacou

CostacouT@edc.pitt.edu

1 Department of Epidemiology, University of Pittsburgh, Pittsburgh, PA, USA

\section{Introduction}

Cardiovascular disease is the leading cause of mortality in individuals with type 1 diabetes [1]. CHD, the most prevalent form of CVD, develops at an earlier age and is disproportionately 


\section{Research in context}

\section{What is already known about this subject?}

- $\quad$ Type 1 diabetes is associated with greater CHD risk

- Having a more favourable American Heart Association-defined cardiovascular health metrics profile is associated with lower CVD risk in the general population

\section{What is the key question?}

- What is the association between the American Heart Association cardiovascular health metrics and CHD development in individuals with type 1 diabetes?

What are the new findings?

- A majority of young adults with type 1 diabetes initially met the ideal criteria for BMI, smoking, blood pressure and total cholesterol, while a lower percentage met the ideal criteria for glucose control and diet

- Each additional ideal cardiovascular health metric was associated with a $19 \%$ lower risk, and each unit increase in total cardiovascular health metric score was associated with a 17\% lower risk of CHD development over 25 years of follow-up, adjusting for kidney disease/function, diabetes duration, depression, triacylglycerol and white blood cell count

\section{How might this impact on clinical practice in the foreseeable future?}

- These findings indicate that small differences in the straightforward American Heart Association cardiovascular health metrics in early adulthood are associated with lower risk for CHD among individuals with type 1 diabetes during 25 years of follow-up, providing a promising framework for future prevention efforts

burdensome among individuals with type 1 diabetes compared with the general population $[2,3]$. Since the incidence of type 1 diabetes is increasing worldwide [4] without a known intervention that is effective in preventing it, measures to delay or decrease diabetes complications are essential. Early prevention of CHD to offset earlier development and greater prevalence with ageing $[5,6]$ has therefore become a priority.

In 2010, the American Heart Association (AHA) created a framework for assessing and promoting cardiovascular health using seven simple metrics, known as Life's Simple Seven (LS7). These metrics include four 'health behaviours' (smoking, BMI, physical activity and diet) and three 'health factors' (total cholesterol, blood pressure and glucose) [7]. The AHA also created ideal, intermediate and poor categories for each metric using criteria in line with clinical practice and public health guidelines [7]. Longitudinal studies demonstrated an inverse linear dose-response relationship between a greater number of metrics within the ideal range and cardiovascular mortality [8, 9] and CVD development over time [10, 11]. LS7 is meant to reduce CVD burden by offering a straightforward, simple way to quantify important health behaviours and health factors to provide clear, actionable change goals. This framework has potential utility in public health messaging and as an assessment and goal-setting tool in clinical practice [12].

The composite of modifiable metrics that compose LS7 has not yet been explored in relation to CHD in the type 1 diabetes population. Traditional risk factors such as blood pressure, blood lipids, waist-to-hip ratio and smoking have been shown to be related to CHD risk in type 1 diabetes, while population-specific risk factors such as diabetes duration and overt nephropathy also influence risk [13-16]. A small body of research examined the prevalence of ideal cardiovascular health metrics in type 1 diabetes $[17,18]$ and found an association between cardiovascular health and development of coronary artery calcium [18], although the prospective association between the LS7 metrics and CHD development has not yet been determined.

The Pittsburgh Epidemiology of Diabetes Complications (EDC) study is a prospective study of individuals with childhood-onset type 1 diabetes with over 25 years of follow-up. The availability of extensive longitudinal data in this cohort allowed for the opportunity to describe baseline measures of ideal, intermediate and poor cardiovascular health metrics based on LS7, and to determine the association between these cardiovascular health metrics and future CHD. It was hypothesised that a greater number of baseline cardiovascular health metrics within the ideal range and a higher total cardiovascular health metric score would be associated with lower CHD incidence.

\section{Methods}

\section{Study design and participants}

EDC study participants were identified based on clinical diagnosis of type 1 diabetes before the age of 17 between 1950 and 
1980 at the Children's Hospital of Pittsburgh [19]. A total of 658 participants were seen at the baseline visit (1986-1988) and have been prospectively followed through clinic examinations occurring biennially for the first 10 years and at years 18,25 and 30 . In addition, surveys were conducted biennially throughout the study. Outcome data collected through the 2013-2015 (25 year) visit were used for this analysis. As separate LS7 cut-points were developed for use in adults (defined as $\geq 20$ years of age by the AHA) and children, participants $<20$ years of age at baseline risk-factor assessment were excluded ( $n=111,12$ of whom developed CHD during follow-up), as were those with prevalent CHD by the second visit (years 1988-1990, $n=68$ ). Thus, the total eligible sample for this analysis was 479 individuals. All participants provided written, informed consent and all protocols were approved by the Institutional Review Board at the University of Pittsburgh.

\section{Cardiovascular health metrics}

Components (i.e., health behaviours and health factors) of the cardiovascular health metrics scores were based on the mean of measures taken at the first and second visits to provide more robust estimates. If a measure was only available at one visit, then that value was used. Participants with missing data at both visits for any variable were excluded. Categories of ideal, intermediate and poor cardiovascular health metrics were defined using AHA criteria [7], with some necessary modifications as indicated below and shown in Table 1.

\section{Four 'health behaviours'}

\section{1) $B M I$}

BMI $\left(\mathrm{kg} / \mathrm{m}^{2}\right)$ was calculated using height and weight measured at clinic visits. The ideal BMI range was defined as $<25$, intermediate as $25-30$ and poor as $\geq 30 \mathrm{~kg} / \mathrm{m}^{2}$.

\section{2) Smoking}

Smoking status was self-reported using a demographic/ medical history questionnaire. In this analysis, consistent with the approach taken previously [17], ideal was defined as 'never smoker', intermediate as 'former smoker' and poor as 'current smoker'. 'Never smokers' were defined as those who stated at both the first and second visits that they had never smoked 100 or more cigarettes. 'Former smokers' indicated that they were former smokers at both visits or that they were smokers at the first visit and no longer smoked at the second visit. 'Current smokers' indicated current smoker status at the second visit.

\section{3) Physical activity}

Physical activity status was assessed using the Paffenbarger Physical Activity Questionnaire [20], whose validity in assessing physical activity of moderate or greater (moderate+) intensity was previously shown [21]. The following question was used to determine minutes of activity per week: 'List any sports or recreation you have participated in during the past week. Please include only the time you were physically active', which then included prompts for time and frequency. An added question to the Paffenbarger Questionnaire asked whether the reported level of activity over the past week reflected the participant's usual activity. Activity intensity was determined using metabolic equivalents of task (METs) according to the 2011 Compendium of Physical Activity [22]. Activities of $\geq 3.0$ METs were considered moderate+ and counted toward activity time per week, in line with the AHA criteria for this metric [7]. The ideal range for physical activity was defined as $\geq 150$ min per week of activity of moderate+ intensity. Intermediate was defined as 1-149 min per week of activity of moderate+ intensity and poor as no physical activity.

\section{4) Nutrition}

Diet was assessed using the Harvard/Willet Food Frequency Questionnaire, which was shown to be a valid method for collecting nutrient intake data [23]. Participants indicated how frequently 116 food/drink items were consumed on average over the past year. Questionnaires were optically processed at the Harvard Medical School (Channing Laboratory, Boston, MA, USA) to produce daily nutrient consumption data. Unfortunately, food group data were not available. Nutrient components included in determining cardiovascular health metrics scores were chosen from a consensus among the rationale for the AHA LS7, Dietary Reference Intakes and ADA recommendations for dietary intake for people with diabetes. Components of the score include: (1) sodium intake of $<2300 \mathrm{mg} / \mathrm{day}$; (2) saturated fat intake of $<10 \%$ of total calories; and (3) fibre intake of $\geq 25 \mathrm{~g} /$ day for women and $38 \mathrm{~g} /$ day for men. The sodium criterion is consistent with the ADA recommendations for individuals with diabetes and Dietary Reference Intake [24, 25]. The fibre criteria are intended to reflect ADA and AHA LS7 recommendations for greater intake of whole grains, fruits and vegetables [7, 24], with sex-specific intake cutpoints based on Dietary Reference Intake values [25]. The saturated fat criterion is explicit in ADA guidelines for recommended intake for individuals with diabetes, and restricting saturated fat intake is mentioned in the LS7 strategic plan [7, 24]. Ideal status was defined as meeting all three criteria, intermediate as meeting two and poor as meeting one or none.

\section{Three 'health factors'}

\section{1) Total cholesterol}

Serum total cholesterol was determined enzymatically from blood drawn after fasting [26]. Per AHA LS7 ranges, ideal total cholesterol was defined as $<5.18 \mathrm{mmol} / 1$, intermediate as $5.18-6.21$ or treated to ideal range and poor as $\geq 6.22$ [7]. Lipid medication use, which was self-reported using a 
Table 1 Cardiovascular health metrics categories

\begin{tabular}{|c|c|c|c|}
\hline Metric & Ideal & Intermediate & Poor \\
\hline Total cholesterol & $<5.18 \mathrm{mmol} / 1$ & $5.18-6.21 \mathrm{mmol}$ or treated to the ideal range & $\geq 6.22 \mathrm{mmol} / 1$ \\
\hline Blood pressure & $<120 \mathrm{mmHg}$ systolic, $<80 \mathrm{mmHg}$ diastolic & $\begin{array}{l}120-139 \mathrm{mmHg} \text { systolic or } 80-89 \mathrm{mmHg} \\
\text { diastolic or treated to the ideal range }\end{array}$ & $\begin{array}{l}\geq 140 \mathrm{mmHg} \text { systolic } \\
\quad \text { or } \geq 90 \mathrm{mmHg} \text { diastolic }\end{array}$ \\
\hline $\mathrm{HbA}_{1 \mathrm{c}}$ & $\begin{array}{l}<53 \mathrm{mmol} / \mathrm{mol} \\
(<7 \%)\end{array}$ & $\begin{array}{l}53-75 \mathrm{mmol} / \mathrm{mol} \\
(7.0-8.9 \%)\end{array}$ & $\begin{array}{l}\geq 75 \mathrm{mmol} / \mathrm{mol} \\
(>9 \%)\end{array}$ \\
\hline BMI & $<25 \mathrm{~kg} / \mathrm{m}^{2}$ & $25-29.9 \mathrm{~kg} / \mathrm{m}^{2}$ & $\geq 30 \mathrm{~kg} / \mathrm{m}^{2}$ \\
\hline Smoking status & Never & Former & Current \\
\hline Physical activity & $\geq 150$ min per week moderate+ & 1-149 min per week moderate+ & None \\
\hline Nutrition & $\begin{array}{l}3 \text { components: } \\
\text { (1) Fibre intake }>25 \mathrm{~g} \text { women, }>38 \mathrm{~g} \text { men } \\
\text { (2) Sodium intake }<2300 \mathrm{mg} \\
\text { (3) Percentage of calories from } \\
\text { saturated fat }<10 \%\end{array}$ & 2 components & $0-1$ component \\
\hline
\end{tabular}

Ideal metric scores: sum count for each participant of metrics in the ideal range (possible score 0-7)

Total metric scores: sum of each participant's metrics across all ranges where ideal $=2$, intermediate $=1$, poor $=0$ (possible score $0-14)$

medical history questionnaire, at either first or second visit was considered when determining categories.

\section{2) Blood pressure}

Blood pressure was measured with a random zero sphygmomanometer; the mean of the last two of three readings taken after a $5 \mathrm{~min}$ rest was used. In accordance with the AHA LS7 status ranges, ideal blood pressure was defined as systolic blood pressure (SBP) $<120 \mathrm{mmHg}$ and diastolic blood pressure (DBP) $<80 \mathrm{mmHg}$, intermediate as SBP $120-139$ or DBP $80-89$ or treated to ideal range, and poor as $\mathrm{SBP} \geq 140$ or $\mathrm{DBP} \geq 90$ [7]. Self-reported antihypertensive medication use at either visit was considered when determining categories.

\section{3) $\mathrm{HbA}_{1 c}$}

$\mathrm{HbA}_{1 \mathrm{c}}$, a more appropriate indicator of overall glucose management in the type 1 diabetes population, was used instead of the LS7 metric of fasting blood glucose. Stable $\mathrm{HbA}_{1}$ was measured by ion-exchange chromatography (Isolab, Akron, OH, USA) for the first 18 months of EDC, and subsequently by automated high-performance liquid chromatography (Diamat; Bio-Rad, Hercules, CA, USA). The two assays were highly correlated $(r=0.95) . \mathrm{HbA}_{1}$ values were converted to DCCT-aligned $\mathrm{HbA}_{1 \mathrm{c}}$ values using a regression equation derived from duplicate assays [DCCT $\mathrm{HbA}_{1 \mathrm{c}}=$ $\left.0.14+0.83\left(\mathrm{EDC}^{\mathrm{HbA}} \mathrm{A}_{1}\right)\right]$. Consistent with the current $\mathrm{ADA}$ guidelines for glycaemic targets, the ideal range for $\mathrm{HbA}_{1 \mathrm{c}}$ was $<53 \mathrm{mmol} / \mathrm{mol}(<7 \%)$ [27], intermediate was $53-$ $75 \mathrm{mmol} / \mathrm{mol}(7-8.9 \%)$ and poor was $\geq 75 \mathrm{mmol} / \mathrm{mol}(\geq 9 \%)$.

\section{CHD outcomes}

The primary outcome of interest was time to development of CHD, defined as the first instance of CHD death, myocardial infarction confirmed by Q-waves on electrocardiogram
(Minnesota codes 1.1 or 1.2) or hospital records, angiographic stenosis $\geq 50 \%$, revascularisation, ischaemic electrocardiogram changes (Minnesota codes 1.3, 4.1-4.3, 5.1-5.3, 7.1) or EDC study physician-diagnosed angina. Self-reported CHD events were confirmed using medical records. CHD as either the primary cause or a contributing cause of death was determined using death certificates, autopsy reports and coroner reports and was reviewed in accordance with the Diabetes Epidemiology Research International mortality protocol [28]. Secondary outcomes of hard CHD (defined as CHD, but excluding angina and ischaemia) and major adverse cardiovascular events (MACE, defined as the first instance of myocardial infarction, stroke or cardiovascular mortality) were also examined.

\section{Other measures of interest}

Potential confounding variables were considered based on previous research looking at CHD $[13,14]$ and cardiovascular health metrics $[17,18]$ in type 1 diabetes. Duration of diabetes was determined using date of diagnosis. Sex, race/ethnicity and household income were specified in a demographic questionnaire administered at first visit. All additional covariates used the mean of first and second visit measures. The Beck Depression Inventory (BDI) questionnaire was used to ascertain depressive symptoms [29, 30]. Triacylglycerols were obtained enzymatically using fasting blood draw [19, 31]. White blood cell count (WBC) was measured using a Counter model S-plus IV (Coulter Electronics, Hialeah, FL, USA). Urinary albumin was measured by immunonephelometry in three timed urines [32]. eGFR was calculated using the Chronic Kidney Disease Epidemiology Collaboration creatinine equation [33]. 


\section{Analysis}

Cardiovascular health metrics were quantified as 'ideal score', indicating the sum of the seven metrics within the ideal range (possible range $0-7$ ). In addition, a 'total score' was determined representing the sum across all seven metrics, where each metric with poor status was assigned a value of 0 , intermediate a value of 1 and ideal a value of 2 (possible range 0 14). Ideal score and total score were used as continuous predictors of time from cardiovascular health metric assessment (i.e. starting from the date of the second clinic visit) to first CHD event using Cox proportional hazard models. Models were adjusted for potential confounders, including sex, diabetes duration at visit 1 and the mean of visit 1 and 2 triacylglycerols, BDI, WBC, household income, AER and eGFR. AER and triacylglycerols were log transformed and quartiles of BDI were used to reduce skewness. Covariates were included based on stepwise selection at the level of $p<0.25$ for entry and $p<0.15$ to retain.

As renal disease is a strong risk factor for CHD in type 1 diabetes, effect modification of the association between total metric score and CHD by eGFR was assessed by including a metric score $\times$ eGFR interaction term in Cox models. All Cox models were confirmed to meet the proportional hazards assumption by assessing that interaction terms between the metric score variables and time were not statistically significant. Additional analyses evaluated ideal and total 'health behaviour' and 'health factor' scores separately as predictors of CHD, hard CHD and MACE outcomes. Two sensitivity analyses were conducted: (1) including repeated assessments of lipid and blood pressure medication use during follow-up in models; and (2) including only participants who indicated that their reported activity reflected their usual activity level. Absolute risk was calculated using Kaplan-Meier survival probabilities for high vs low scores based on the score midpoint ( $>3$ vs $\leq 3$ ideal and $>7$ vs $\leq 7$ total). All analyses were conducted using SAS version 9.4 (SAS Institute, Cary, NC, USA).

\section{Results}

Of 479 eligible participants, 43 were excluded due to missing cardiovascular health metric or covariate data and one had unknown CHD status through 25 years of follow-up, leaving a final sample of $435(90.8 \%)$. Median participant age at the first visit was 28.6 years, median diabetes duration was 19.8 years, men and women were evenly represented and the sample was $97.9 \%$ white (Table 2). At baseline, three participants reported taking lipid medications and 57 reported taking blood pressure medications. During the 25 years of follow-up, 177 participants (40.7\%) had an incident CHD event (fatal CHD: 18; non-fatal myocardial infarction: 48;
Table 2 Participant characteristics (mean of first visit [1986-1988] and second visit [1988-1990]; $n=435$ )

\begin{tabular}{|c|c|}
\hline Characteristic & $\begin{array}{l}\text { Median (25th and } 75 \text { th } \\
\text { percentiles) or } N(\%)\end{array}$ \\
\hline Age (years, at first visit) ${ }^{\mathrm{a}}$ & $28.6(24.5,33.3)$ \\
\hline Diabetes duration (years, at first visit) ${ }^{\mathrm{a}}$ & $19.8(15.2,26.0)$ \\
\hline Sex (male) & $217(49.9 \%)$ \\
\hline Triacylglycerols (mmol/l) & $0.94(0.69,1.39)$ \\
\hline Total cholesterol (mmol/l) & $4.78(4.29,5.56)$ \\
\hline HDL-cholesterol $(\mathrm{mmol} / \mathrm{l}) ; n=434$ & $1.35(1.17,1.57)$ \\
\hline LDL-cholesterol $(\mathrm{mmol} / \mathrm{l}) ; n=428$ & $2.93(2.46,3.50)$ \\
\hline Race: white & $426(97.9 \%)$ \\
\hline $\operatorname{AER}(\mu \mathrm{g} / \mathrm{min})$ & $18.3(8.3,213.2)$ \\
\hline $\mathrm{eGFR}\left(\mathrm{ml} \mathrm{min}{ }^{-1} 1.73 \mathrm{~m}^{-2}\right)$ & $109.5(91.6,121.0)$ \\
\hline Blood pressure $(\mathrm{mm} / \mathrm{Hg})$ & $\begin{array}{l}112.5(105.0,122.0) / 72.5 \\
\quad(66.5,79.0)\end{array}$ \\
\hline \multicolumn{2}{|l|}{$\mathrm{HbA}_{1 \mathrm{c}}$} \\
\hline $\mathrm{mmol} / \mathrm{mol}$ & $67(62,81)$ \\
\hline$\%$ & $8.7(7.8,9.6)$ \\
\hline WBC $\left(10^{9} / 1\right)$ & $6.4(5.6,7.7)$ \\
\hline BMI $\left(\mathrm{kg} / \mathrm{m}^{2}\right)$ & $23.9(21.8,25.8)$ \\
\hline Physical activity (min/week moderate+) & $90(0,255)$ \\
\hline BDI & $5.5(2.5,9.5)$ \\
\hline
\end{tabular}

${ }^{a}$ Only baseline data used for age and diabetes duration

revascularisation: 36; ischaemic electrocardiogram: 25; angina: 50).

At baseline (Fig. 1), the majority of participants were in the ideal range for total cholesterol $(64.4 \%)$, blood pressure (64.4\%), BMI (64.6\%) and smoking status (58.2\%). Only $7.4 \%$ had ideal $\mathrm{HbA}_{1 \mathrm{c}}$ and $1.2 \%$ had ideal nutrition component status. The ideal physical activity goal was met by $39.4 \%$ of participants.

The median number of ideal metrics was 3 (IQR: 2-4; minimum: 0, maximum: 6). The median total metrics score was 8 (IQR: 6-9; minimum: 2, maximum: 13 ). No participants achieved ideal status for all seven metrics.

\section{Ideal metric score}

In unadjusted models, each additional ideal metric was associated with a $38 \%$ decreased risk of CHD $(p<0.01)$ (Table 3$)$. This association was attenuated to $32 \%(p<0.01)$ adjusting for diabetes duration; it was further attenuated to $24 \%$ $(p<0.01)$ with additional adjustment for eGFR and AER; and to $19 \%(p=0.01)$ in the final multivariable model adding triacylglycerols, WBC and depression (no significant eGFR effect modification was found). Each additional ideal 'health factor' metric was associated with a $26 \%$ decreased risk $(p<0.01)$ in final models with adjustment for the same 
Fig. 1 Prevalence of cardiovascular health metrics (mean of first visit [1986-1988] and second visit [1988-1990]; $n=435$ ). Prevalence estimates are the mean of measures taken at the first and second visits. If a measure was missing at one of the two visits, the measure from the other visit was used

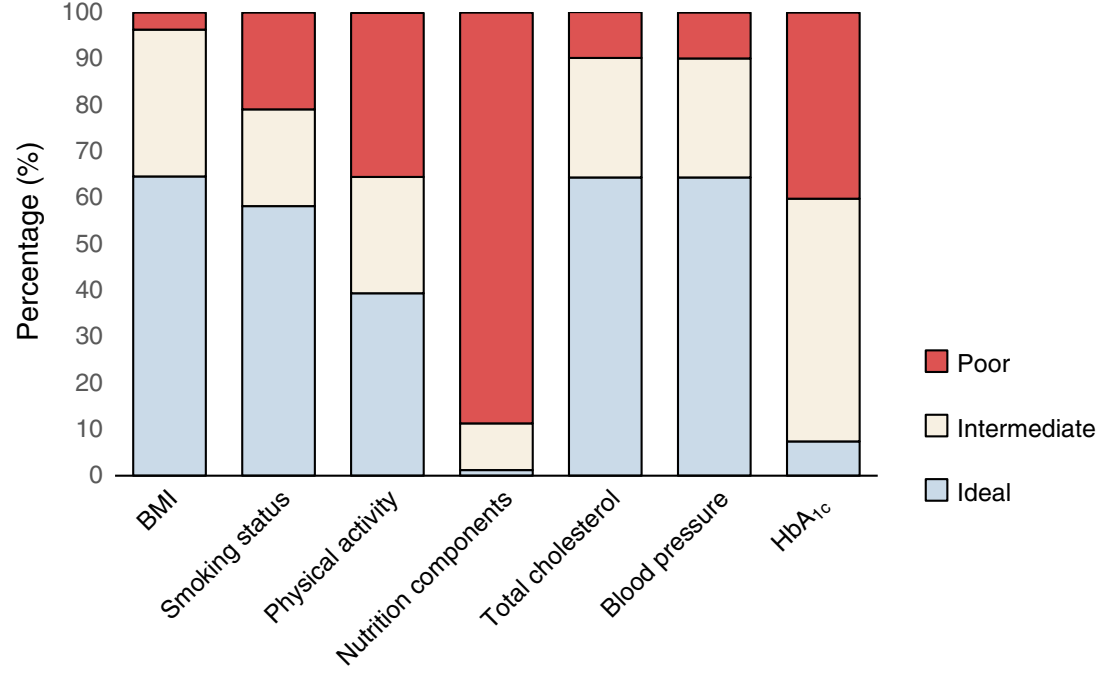

Cardiovascular health metric covariates as the full score plus cardiovascular health behaviours. Risk reduction with each additional ideal health behaviour was significant in unadjusted models (HR: $0.68, p<0.01$ ) and no longer significant in fully adjusted models.

\section{Total metric score}

Each unit increase in total cardiovascular health metric score was associated with a $27 \%$ decreased risk of CHD $(p<0.01)$, which was attenuated to $26 \%(p<0.01)$ in models adjusted for diabetes duration alone; $21 \%(p<0.01)$ when adjusting for diabetes duration, eGFR and AER; and 17\% in final models with additional adjustment for triacylglycerols, WBC and BDI (Table 3). Each unit increase in total 'health behaviour' score was associated with a $13 \%$ decreased adjusted risk of CHD $(p=0.04)$, and each unit increase in total 'health factor' score with a $21 \%$ decreased risk $(p<0.01)$.

Similar results were obtained when examining hard CHD (i.e. excluding angina and ischaemic electrocardiogram, $n=$ 475 , events $=166$; Table 3 ). Results were also similar with

Table 3 Cox proportional hazards outcome analysis of ideal score or total score and CHD, hard CHD and MACE as HR (95\% CI)

\begin{tabular}{|c|c|c|c|c|c|c|c|c|}
\hline \multirow[t]{2}{*}{ Variable } & \multicolumn{8}{|c|}{ Cox proportional HR } \\
\hline & Unadjusted & $p$ value & $\begin{array}{l}\text { Adjusted for } \\
\text { diabetes } \\
\text { duration }\end{array}$ & $p$ value & $\begin{array}{l}\text { Adjusted for } \\
\text { duration, } \\
\text { AER, log eGFR }\end{array}$ & $p$ value & Fully adjusted $^{\mathrm{a}}$ & $p$ value \\
\hline \multicolumn{9}{|l|}{ Total CHD $(n=435$, events $=177)$} \\
\hline Ideal score & $0.62(0.55,0.71)$ & $<0.01$ & $0.68(0.60,0.77)$ & $<0.01$ & $0.76(0.66,0.87)$ & $<0.01$ & $0.81(0.70,0.94)$ & 0.01 \\
\hline Ideal 'health behaviours' only ${ }^{\mathrm{b}}$ & $0.68(0.54,0.86)$ & $<0.01$ & $0.76(0.61,0.96)$ & 0.02 & $0.79(0.62,1.00)$ & 0.05 & $0.88(0.69,1.12)$ & 0.28 \\
\hline Ideal 'health factors' only ${ }^{\mathrm{b}}$ & $0.60(0.52,0.70)$ & $<0.01$ & $0.65(0.56,0.76)$ & $<0.01$ & $0.75(0.64,0.88)$ & $<0.01$ & $0.78(0.66,0.93)$ & 0.01 \\
\hline Total score & $0.73(0.67,0.79)$ & $<0.01$ & $0.74(0.69,0.81)$ & $<0.01$ & $0.79(0.72,0.86)$ & $<0.01$ & $0.83(0.75,0.92)$ & $<0.01$ \\
\hline Total 'health behaviours' only ${ }^{\mathrm{b}}$ & $0.76(0.67,0.86)$ & $<0.01$ & $0.80(0.71,0.91)$ & $<0.01$ & $0.82(0.72,0.93)$ & $<0.01$ & $0.87(0.76,0.99)$ & 0.04 \\
\hline Total 'health factors' only ${ }^{\mathrm{b}}$ & $0.70(0.64,0.77)$ & $<0.01$ & $0.71(0.64,0.78)$ & $<0.01$ & $0.77(0.68,0.86)$ & $<0.01$ & $0.79(0.70,0.90)$ & $<0.01$ \\
\hline \multicolumn{9}{|c|}{ Hard CHD $(n=475$ [92\% of 519 eligible], events $=166)$} \\
\hline Ideal score & $0.59(0.51,0.67)$ & $<0.01$ & $0.65(0.57,0.75)$ & $<0.01$ & $0.75(0.64,0.87)$ & $<0.01$ & $0.80(0.68,0.93)$ & 0.01 \\
\hline Total score & $0.69(0.64,0.75)$ & $<0.01$ & $0.72(0.67,0.79)$ & $<0.01$ & $0.78(0.72,0.86)$ & $<0.01$ & $0.82(0.74,0.90)$ & $<0.01$ \\
\hline \multicolumn{9}{|c|}{ MACE $(n=477$ [92\% of 518 eligible], events $=133)$} \\
\hline Ideal score & $0.52(0.45,0.61)$ & $<0.01$ & $0.59(0.51,0.68)$ & $<0.01$ & $0.67(0.57,0.79)$ & $<0.01$ & $0.74(0.62,0.88)$ & $<0.01$ \\
\hline Total score & $0.68(0.62,0.74)$ & $<0.01$ & $0.72(0.66,0.79)$ & $<0.01$ & $0.78(0.71,0.86)$ & $<0.01$ & $0.85(0.77,0.94)$ & $<0.01$ \\
\hline
\end{tabular}

Participants censored at time of first event or at last follow-up

${ }^{a}$ Adjusted for duration of diabetes, AER, eGFR, triacylglycerols, depression and WBC

${ }^{\mathrm{b}}$ Adjusted for factors or behaviours 
MACE as the outcome of interest $(n=477$, events $=133)$, with a slightly stronger $26 \%$ decreased risk $(p<0.01)$ associated with each unit increase in ideal metric score in the fully adjusted model. Sensitivity analysis found no difference in time-to-event models when including repeated assessments of medication use, which increased over time (electronic supplementary material [ESM] Table 1), and, separately, when restricted to those who reported that their activity level reflected their usual level of activity $(n=368)$. After 25 years, the absolute risk reduction for total CHD for high vs low ideal scores $(>3$ vs $\leq 3)$ was $0.498(95 \%$ CI $0.415,0.581)$ and for high vs low total metric scores (>7 vs $\leq 7$ ) was 0.153 (95\% CI $0.122,0.184)$.

\section{Discussion}

A carefully designed longitudinal study of a large cohort of individuals with childhood-onset type 1 diabetes allowed for the examination of the relationship between measures of a modified version of the AHA's cardiovascular health metrics in early adulthood and incident CHD. Each additional cardiovascular health metric within the ideal range and each unit increase in total metric score was associated with a lower incidence of CHD (19\%, $p=0.01$ and 17\%, $p<0.01$, respectively) over a 25 year follow-up. These findings support the hypothesis that a more favourable cardiovascular health metrics profile in early adulthood is associated with a lower incidence of CHD in type 1 diabetes, showing the potential utility of the AHA LS7 as a tool to identify and promote improvement in health behaviours and health factors in this population.

This is the first known report of the association between cardiovascular health metrics and incident CHD in type 1 diabetes and was done in the EDC study with data on all seven metrics over 25 years of follow-up. Measures from the first two clinic assessment visits (young adulthood) were considered for all metrics as the time of exposure of interest due to risk for earlier development of CHD among individuals with type 1 diabetes. Granted, physical activity and nutrient intake were self-reported and diet could not be characterised using the same criteria outlined by LS7 due to the availability of nutrient intake data. Also, repeated measures of diet data were limited, not allowing the assessment of repeated measures of the full LS7 profile.

While the EDC cohort is representative of baseline type 1 diabetes prevalence in the geographic area at the time [34], the lack of racial/ethnic diversity is a limitation in this study, especially given an increased incidence among black individuals [35].

The current analysis builds on existing literature exploring the prevalence of cardiovascular health metrics in individuals with type 1 diabetes. In comparison with the EDC cohort, the Type 1 Diabetes Exchange clinic registry (years 2010-2012) and the Coronary Artery Calcification in Type 1 Diabetes (CACTI, years 2000-2002) cohort found that a greater percentage of their study samples met the $\mathrm{HbA}_{1 \mathrm{c}}$ goal, but a lower percentage met the ideal criteria for blood pressure and BMI $[17,18]$. The CACTI study also demonstrated a similar prevalence of individuals meeting the ideal criteria for diet, total cholesterol and smoking and a lower prevalence of ideal physical activity when compared with the EDC [17]. Differences in prevalence may be due to an older mean age of 37 years in both the Diabetes Exchange and CACTI cohorts compared with the EDC cohort, differences in years when measures were taken (1986-1990 vs 2000-2002 and 2010 2012) $[17,18]$ and differences in activity assessment across studies [17]. In addition, EDC participants were not directly treated by research staff, while 'Exchange' members were seen in the reporting clinics and about half of the CACTI participants were treated by study personnel. The lack of guidelines for blood glucose management and dietary intake among the type 1 population in the 1986-1990 time period may have further contributed to lower prevalence of ideal $\mathrm{HbA}_{1 \mathrm{c}}$ and diet in the EDC cohort. However, more contemporary estimates in type 1 diabetes youth in the SEARCH for Diabetes in Youth study [36] and adults in the 'Exchange' (years 2016-2018) [37] have demonstrated similar mean levels of $\mathrm{HbA}_{1 \mathrm{c}}$ compared with similarly aged EDC participants in the mid/late 1980s. Also, the comparably low prevalence of ideal diet in CACTI is noteworthy, indicating that perhaps components of the risk profile have remained somewhat unchanged over time.

There are inconsistencies in studies of cardiovascular health metric prevalence in the general population compared with those among individuals with type 1 diabetes. The Framingham Offspring study from a similar time period to EDC demonstrated a comparatively lower prevalence vs EDC of ideal smoking status, total cholesterol and blood pressure [10]. National Health and Nutrition Examination Survey (NHANES) metric estimates among a comparable age range to EDC baseline also demonstrated lower prevalence of ideal BMI [38]. In contrast, the CACTI study, which compared all seven cardiovascular health metrics in a type 1 diabetes population with individuals without diabetes, found that blood pressure was significantly less favourable among the former [17]. In summary, CACTI found less favourable while EDC found more favourable metrics profiles in their cohorts with type 1 diabetes compared with individuals without diabetes. Possible reasons for these discrepancies include the fact that the CACTI study was initiated over 15 years after EDC baseline and involves a control group composed primarily of family and friends of enrolled individuals with type 1 diabetes.

Longitudinal examination of cardiovascular health metrics and future atherosclerotic outcomes in type 1 diabetes has only been done previously in the CACTI study, which found that a higher number of ideal cardiovascular health metrics 
was associated with decreased coronary artery calcium progression [17]. The current analysis from the EDC study demonstrated that each unit increase in ideal and total cardiovascular health metric scores was associated with a lower risk for CHD and MACE. This EDC study also looked at 'health behaviours' and 'health factors' as independent predictors of CHD. Ideal health behaviours were not associated with CHD in fully adjusted models, which may speak to the influence of depression on health behaviour and the overall health profile of participants in the ideal range for BMI. Also, it is important to note that adjustment for post-baseline lipid medication use did not eliminate the value of LS7 as a predictor of CHD in the EDC. This further underscores the utility of LS7 as a predictor, given that young adults with 20 years of type 1 diabetes duration commonly reach risk levels meriting statin use under current guidelines [5].

Our findings in the EDC cohort are in line with findings in the general population demonstrating a dose-response relationship between the number of ideal cardiovascular health metrics and CVD risk. In the Framingham Offspring study, for each unit increase in the ideal cardiovascular health metric score (range 0-7), CVD incidence decreased by 23\% [39]. Similarly, others reported that achieving five or more ideal metrics was associated with a significantly lower CVD risk compared with zero or one ideal metric [11, 40]. Achieving the highest total cardiovascular health metric score, quantified in the same way as in the EDC study, significantly reduced CVD risk [41, 42]. Finally, in the limited literature looking at CHD in the general population, self-reported CHD has been shown to be lower among individuals with more ideal cardiovascular health metrics [43].

The fact that a similar or more favourable cardiovascular health risk profile in type 1 diabetes compared with the general population occurs despite a relatively higher burden of CHD, as shown in the EDC, warrants further consideration. For example, previous EDC findings indicate that treatment guidelines may need to consider lower blood pressure goals in individuals with type 1 diabetes in order to decrease CHD risk [44]. While the current analysis was intended to evaluate the AHA LS7 criteria with only minimal necessary changes, future analyses could consider additional modifications to create a more population-specific LS7.

A potential limitation to the LS7 approach is that it does not provide information on the relative importance of each component and is not meant to serve as a risk prediction model. Consistent with other studies [9, 41], the current findings indicate that any improvement in LS7 metrics scores is associated with decreased CVD risk. Future research can investigate the utilisation of LS7 in children and further explore the relative importance of CVD risk factors and resulting LS7 metrics in the type 1 diabetes population [45]. Additional studies of the impact of interventions designed to improve cardiovascular health on LS7 are also needed.
In conclusion, cardiovascular health metrics show promise for use in type 1 diabetes as a clinical and public health promotion tool and provide insight into areas of greatest need of intervention to improve cardiovascular health in specific type 1 diabetes cohorts. In the EDC cohort, diet had an ideal prevalence of $1.2 \%$, making it a first-line intervention target. Extending the application of the LS7 approach, which allows for easy assessment and sets straightforward goals for modifiable risk factors, to the high-risk type 1 diabetes population has great potential to promote cardiovascular health.

Supplementary Information The online version contains peer-reviewed but unedited supplementary material available at https://doi.org/10.1007/ s00125-020-05328-9.

Acknowledgements The authors thank the participants in the Pittsburgh Epidemiology of Diabetes Complications study and the EDC study personnel for their support and contributions. Graphical abstract created with BioRender.com

Data availability The datasets analysed for this study are available from the corresponding author on reasonable request. Data from this manuscript were presented at the 2019 American Heart Association Epidemiology/Lifestyle conference.

Funding The EDC was supported by NIH grant DK34818 and the Rossi Memorial Fund. SMD's contributions were additionally supported by the NHLBI NIH T32HL083825 training grant.

Authors' relationships and activities The authors declare that there are no relationships or activities that might bias, or be perceived to bias, their work.

Contribution statement TC, TJO and RGM contributed to the EDC study design and data acquisition. SMD contributed to the design of this analysis, conducted data analysis, wrote the manuscript and gave final approval of the version to be published. TC, TJO, RGM and AMK assisted with analysis and interpretation of the data, reviewed the manuscript for intellectual content and gave final approval of the version to be published. TC is the guarantor of this work.

\section{References}

1. Secrest AM, Becker DJ, Kelsey SF, Laporte RE, Orchard TJ (2010) Cause-specific mortality trends in a large population-based cohort with long-standing childhood-onset type 1 diabetes. Diabetes 59(12):3216-3222. https://doi.org/10.2337/db10-0862

2. de Ferranti SD, de Boer IH, Fonseca V et al (2014) Type 1 diabetes mellitus and cardiovascular disease: a scientific statement from the American Heart Association and American Diabetes Association. Diabetes Care 37(10):2843-2863. https://doi.org/10.2337/dc141720

3. Krolewski AS, Kosinski EJ, Warram JH et al (1987) Magnitude and determinants of coronary artery disease in juvenile-onset, insulindependent diabetes mellitus. Am J Cardiol 59(8):750-755. https:// doi.org/10.1016/0002-9149(87)91086-1

4. International Diabetes Federation (2017) IDF diabetes atlas 2017, 8th edn. International Diabetes Federation, Brussels 
5. Miller RG, Mahajan HD, Costacou T, Sekikawa A, Anderson SJ, Orchard TJ (2016) A contemporary estimate of total mortality and cardiovascular disease risk in young adults with type 1 diabetes: the Pittsburgh Epidemiology of Diabetes Complications Study. Diabetes Care 39(12):2296-2303. https://doi.org/10.2337/dc161162

6. Lee SI, Patel M, Jones CM, Narendran P (2015) Cardiovascular disease and type 1 diabetes: prevalence, prediction and management in an ageing population. Ther Adv Chronic Dis 6(6):347374. https://doi.org/10.1177/2040622315598502

7. Lloyd-Jones DM, Hong Y, Labarthe D et al (2010) Defining and setting national goals for cardiovascular health promotion and disease reduction: the American Heart Association's strategic impact goal through 2020 and beyond. Circulation 121(4):586613. https://doi.org/10.1161/CIRCULATIONAHA.109.192703

8. Yang Q, Cogswell ME, Flanders WD et al (2012) Trends in cardiovascular health metrics and associations with all-cause and CVD mortality among US adults. JAMA 307(12):1273-1283. https://doi. org/10.1001/jama.2012.339

9. Aneni EC, Crippa A, Osondu CU et al (2017) Estimates of mortality benefit from ideal cardiovascular health metrics: a dose response meta-analysis. J Am Heart Assoc 6(12):e006904. https://doi.org/10. 1161/JAHA.117.006904

10. Enserro DM, Vasan RS, Xanthakis V (2018) Twenty-year trends in the American Heart Association Cardiovascular Health Score and impact on subclinical and clinical cardiovascular disease: the Framingham Offspring Study. J Am Heart Assoc 7(11):e008741. https://doi.org/10.1161/JAHA.118.008741

11. Folsom AR, Yatsuya H, Nettleton JA, Lutsey PL, Cushman M, Rosamond WD (2011) Community prevalence of ideal cardiovascular health, by the American Heart Association definition, and relationship with cardiovascular disease incidence. J Am Coll Cardiol 57(16):1690-1696. https://doi.org/10.1016/j.jacc.2010.11. 041

12. Lancarotte I, Nobre MR (2016) Primordial and primary prevention programs for cardiovascular diseases: from risk assessment through risk communication to risk reduction. A review of the literature. Clin (Sao Paulo) 71(11):667-678. https://doi.org/10.6061/clinics/ 2016(11)09

13. Orchard TJ, Olson JC, Erbey JR et al (2003) Insulin resistancerelated factors, but not glycemia, predict coronary artery disease in type 1 diabetes: 10-year follow-up data from the Pittsburgh Epidemiology of Diabetes Complications Study. Diabetes Care 26(5):1374-1379. https://doi.org/10.2337/diacare.26.5.1374

14. Forrest KY, Becker DJ, Kuller LH, Wolfson SK, Orchard TJ (2000) Are predictors of coronary heart disease and lower-extremity arterial disease in type 1 diabetes the same? A prospective study. Atherosclerosis 148(1):159-169. https://doi.org/10.1016/S00219150(99)00217-8

15. Soedamah-Muthu SS, Chaturvedi N, Toeller M et al (2004) Risk factors for coronary heart disease in type 1 diabetic patients in Europe: the EURODIAB Prospective Complications Study. Diabetes Care 27(2):530-537. https://doi.org/10.2337/diacare.27. 2.530

16. Miller RG, Costacou T, Orchard TJ (2019) Risk factor modeling for cardiovascular disease in type 1 diabetes in the Pittsburgh Epidemiology of Diabetes Complications (EDC) study: a comparison with the diabetes control and complications trial/epidemiology of diabetes interventions and complication. Diabetes 68(2):409419. https://doi.org/10.2337/db18-0515

17. Alman AC, Maahs DM, Rewers MJ, Snell-Bergeon JK (2014) Ideal cardiovascular health and the prevalence and progression of coronary artery calcification in adults with and without type 1 diabetes. Diabetes Care 37(2):521-528. https://doi.org/10.2337/ dc13-0997
18. McCarthy MM, Funk M, Grey M (2016) Cardiovascular health in adults with type 1 diabetes. Prev Med 91:138-143. https://doi.org/ 10.1016/j.ypmed.2016.08.019

19. Orchard TJ, Dorman JS, Maser RE et al (1990) Factors associated with avoidance of severe complications after $25 \mathrm{yr}$ of IDDM. Pittsburgh Epidemiology of Diabetes Complications Study I. Diabetes Care 13(7):741-747. https://doi.org/10.2337/diacare.13. 7.741

20. Kriska AM, LaPorte RE, Patrick SL, Kuller LH, Orchard TJ (1991) The association of physical activity and diabetic complications in individuals with insulin-dependent diabetes mellitus: the Epidemiology of Diabetes Complications Study-VII. J Clin Epidemiol 44(11):1207-1214. https://doi.org/10.1016/08954356(91)90153-Z

21. Ainsworth BE, Leon AS, Richardson MT, Jacobs DR, Paffenbarger RSJ (1993) Accuracy of the College Alumnus Physical Activity Questionnaire. J Clin Epidemiol 46(12):1403-1411. https://doi. org/10.1016/0895-4356(93)90140-v

22. Ainsworth BE, Haskell WL, Herrmann SD et al (2011) 2011 compendium of physical activities. Med Sci Sports Exerc 43(8): 1575-1581. https://doi.org/10.1249/MSS.0b013e31821 ece12

23. Willett WC, Sampson L, Stampfer MJ et al (1985) Reproducibility and validity of a semiquantitative food frequency questionnaire. Am J Epidemiol 122(1):51-65. https://doi.org/10.1093/ oxfordjournals.aje.a114086

24. Evert AB, Boucher JL, Cypress M et al (2014) Nutrition therapy recommendations for the management of adults with diabetes. Diabetes Care 37(Suppl 1):S120-S143. https://doi.org/10.2337/ dc14-S120

25. U.S. Department of Health and Human Services and U.S. Department of Agriculture (2015) Dietary guidelines for Americans 2015-2020. In: Appendix 7. Nutritional goals for agesex groups based on dietary reference intakes and dietary guidelines recommendations. Available from https://health.gov/ dietaryguidelines/2015/guidelines/appendix-7/. Accessed 10 July 2018

26. Allain CC, Poon LS, Chan CS, Richmond W, Fu PC (1974) Enzymatic determination of total serum cholesterol. Clin Chem 20(4):470-475. https://doi.org/10.1093/clinchem/20.4.470

27. American Diabetes Association (2018) Position statement 6. Glycemic targets: standards of medical care in diabetes - 2018 . Diabetes Care 41(Suppl 1):S55-S64. https://doi.org/10.2337/ dc18-S006

28. Diabetes Epidemiology Research International Mortality Study Group (1991) International evaluation of cause-specific mortality and IDDM. Diabetes Care 14(1):55-60. https://doi.org/10.2337/ diacare.14.1.55

29. Beck AT, Ward CH, Mendelson M, Mock J, Erbaugh J (1961) An inventory for measuring depression. Arch Gen Psychiatry 4:561571. https://doi.org/10.1001/archpsyc. 1961.01710120031004

30. Beck AT, Steer RA, Carbin MG (1988) Psychometric properties of the Beck Depression Inventory: twenty-five years of evaluation. Clin Psychol Rev 8(1):77-100. https://doi.org/10.1016/02727358(88)90050-5

31. Orchard TJ, Dorman JS, Maser RE et al (1990) Prevalence of complications in IDDM by sex and duration. Pittsburgh Epidemiology of Diabetes Complications Study II. Diabetes 39(9):1116-1124. https://doi.org/10.2337/diab.39.9.1116

32. Ellis D, Buffone GJ (1977) New approach to evaluation of proteinuric states. Clin Chem 23(4):666-670. https://doi.org/10.1093/ clinchem/23.4.666

33. Levey AS, Stevens LA, Schmid CH et al (2009) A new equation to estimate glomerular filtration rate. Ann Intern Med 150(9):604 612. https://doi.org/10.7326/0003-4819-150-9-200905050-00006

34. Wagener DK, Sacks JM, LaPorte RE, Macgregor JM (1982) The Pittsburgh study of insulin-dependent diabetes mellitus. Risk for 
diabetes among relatives of IDDM. Diabetes 31(2):136-144. https://doi.org/10.2337/diab.31.2.136

35. Mayer-Davis EJ, Lawrence JM, Dabelea D et al (2017) Incidence trends of type 1 and type 2 diabetes among youths, 2002-2012. N Engl J Med 376(15):1419-1429. https://doi.org/10.1056/ NEJMoa1610187

36. Jaiswal M, Divers J, Urbina EM et al (2018) Cardiovascular autonomic neuropathy in adolescents and young adults with type 1 and type 2 diabetes: the SEARCH for Diabetes in Youth Cohort Study. Pediatr Diabetes 19(4):680-689. https://doi.org/10.1111/pedi. 12633

37. Foster NC, Beck RW, Miller KM et al (2019) State of type 1 diabetes management and outcomes from the T1D Exchange in 2016-2018. Diabetes Technol Ther 21(2):66-72. https://doi.org/ 10.1089/dia.2018.0384

38. Shay CM, Ning H, Allen NB et al (2012) Status of cardiovascular health in US adults: prevalence estimates from the National Health and Nutrition Examination Surveys (NHANES) 2003-2008. Circulation 125(1):45-56. https://doi.org/10.1161/ CIRCULATIONAHA.111.035733

39. Xanthakis V, Enserro DM, Murabito JM et al (2014) Ideal cardiovascular health: associations with biomarkers and subclinical disease and impact on incidence of cardiovascular disease in the Framingham Offspring Study. Circulation 130(19):1676-1683. https://doi.org/10.1161/CIRCULATIONAHA.114.009273

40. Dong C, Rundek T, Wright CB, Anwar Z, Elkind MSV, Sacco RL (2012) Ideal cardiovascular health predicts lower risks of myocardial infarction, stroke, and vascular death across whites, blacks, and Hispanics. Circulation 125(24):2975-2984. https:// doi.org/10.1161/CIRCULATIONAHA.111.081083

41. Miao C, Bao M, Xing A et al (2015) Cardiovascular health score and the risk of cardiovascular diseases. PLoS One 10(7):e0131537. https://doi.org/10.1371/journal.pone.0131537

42. Polonsky TS, Ning H, Daviglus ML et al (2017) Association of cardiovascular health with subclinical disease and incident events: the multi-ethnic study of atherosclerosis. J Am Heart Assoc 6(3): e004894. https://doi.org/10.1161/JAHA.116.004894

43. Peng Y, Wang Z, Dong B, Cao S, Hu J, Adegbija O (2017) Life's Simple 7 and ischemic heart disease in the general Australian population. PLoS One 12(10):e0187020. https://doi.org/10.1371/ journal.pone. 0187020

44. Guo J, Brooks MM, Muldoon MF, Naimi AI, Orchard TJ, Costacou $\mathrm{T}$ (2019) Optimal blood pressure thresholds for minimal coronary artery disease risk in type 1 diabetes. Diabetes Care 42(9):16921699. https://doi.org/10.2337/dc19-0480

45. Rawshani A, Rawshani A, Sattar N et al (2019) Relative prognostic importance and optimal levels of risk factors for mortality and cardiovascular outcomes in type 1 diabetes mellitus. Circulation 139 (16): $1900-1912$. https://doi.org/10.1161/ CIRCULATIONAHA.118.037454

Publisher's note Springer Nature remains neutral with regard to jurisdictional claims in published maps and institutional affiliations. 